\section{Research Square \\ Preprints are preliminary reports that have not undergone peer review They should not be considered conclusive, used to inform clinical practice, or referenced by the media as validated information.}

\title{
Identification of Differentially Expressed Genes for Disc Degeneration Using Gene Expression Profiling and Bioinformatics Analysis
}

\author{
Ning Fan \\ Beijing Chao-Yang Hospital: Beijing Chaoyang Hospital \\ Shuo Yuan \\ Beijing Chao-Yang Hospital: Beijing Chaoyang Hospital \\ Yong Hai \\ Beijing Chao-Yang Hospital: Beijing Chaoyang Hospital \\ Peng Du \\ Beijing Chao-Yang Hospital: Beijing Chaoyang Hospital \\ Jian Li \\ Beijing Chao-Yang Hospital: Beijing Chaoyang Hospital \\ Xiaochuan Kong \\ Beijing Chao-Yang Hospital: Beijing Chaoyang Hospital \\ Wenyi Zhu \\ Beijing Chao-Yang Hospital: Beijing Chaoyang Hospital \\ Yuzeng Liu \\ Beijing Chao-Yang Hospital: Beijing Chaoyang Hospital \\ Lei Zang ( $\nabla$ zanglei@ccmu.edu.cn ) \\ Beijing Chao-yang Hospital, Capitial medical University https://orcid.org/0000-0003-1403-4159
}

\section{Research article}

Keywords: Disc degeneration, IL-1 $\beta$, Gene expression profiling, Bioinformatics

Posted Date: May 25th, 2021

DOI: https://doi.org/10.21203/rs.3.rs-508157/v1

License: (c) (i) This work is licensed under a Creative Commons Attribution 4.0 International License. Read Full License 


\section{Abstract}

Background

Inflammatory processes exacerbated by IL-1 $\beta$ are believed to be key mediators of disc degeneration and low back pain. However, the underlying mechanism remains unclear. We performed a bioinformatics analysis to identify the key genes that were differentially expressed between degenerative intervertebral disc cells with and without exposure to interleukin (IL)-1 $\beta$, and explore the related signaling pathways and interaction networks.

Methods

The microarray data were downloaded from the Gene Expression Omnibus (GSE 27494). Then, analyses of the gene ontology, signaling pathways, and interaction networks for the differentially expressed genes (DEGs) were conducted using tools including the Database for Annotation, Visualization, and Integrated Discovery (DAVID), Metascape, Gene Set Enrichment Analysis (GSEA),

Search Tool for the Retrieval of Interacting Genes (STRING), Cytoscape, the Venn method, and packages of the R computing language.

Results

A total of 260 DEGs were identified, including 161 upregulated genes and 99 down-regulated genes. Gene Ontology (G0) annotation analysis showed that these DEGs were mainly associated with the extracellular region, chemotaxis, taxis, cytokine activity, and cytokine receptor binding. A Kyoto Encyclopedia of Genes and Genomes (KEGG) signaling pathway analysis showed that these DEGs were mainly involved in the interactions of cytokine-cytokine receptor interaction, rheumatoid arthritis, tumor necrosis factor (TNF) signaling pathway, salmonella infection, and chemokine signaling pathway. The interaction network analysis indicated that 10 hub genes, including CXCL8, CXCL1, CCL20, CXCL2, CXCL5, CXCL3, CXCL6, C3, PF4, and GPER1 may play key roles in intervertebral disc degeneration.

Conclusions

Bioinformatic analysis showed that CXCL8 and other 9 key genes may play a role in the development of disc degeneration induced by inflammatory reactions, and can be used to identify the potential therapeutic target genes.

\section{Background}

Intervertebral disc degeneration (IVDD) is considered to be the leading cause of low back pain, severely threatening the quality of life of middle-aged and elderly people and increasing the economic burden of families and the society $(1,2)$. Although modern evidence-based medicine has identified IVDD as a consequence of a variety of genetic, traumatic, lifestyle, aging, and nutritional factors, the pathological mechanisms involved in IVDD progression remain unknown (3-7). Currently, treatment modalities include noninvasive treatments, such as drug therapy, multiple physical modalities, and multidisciplinary biopsychosocial rehabilitation, interventional modalities, such as intradiscal therapies and epidural injections, regenerative modalities with disc injections of various solutions, and surgical approaches, such as fusion and artificial disc replacement. Despite the progress made in therapies aiming to relieve the pain symptoms, they offer only temporary benefits and are related to complications (8). Therefore, it is necessary to elucidate the underlying pathogenesis of IVDD and lower back pain in order to develop novel effective treatment methods for these conditions.

The role of bioinformatics in the exploration of disease mechanisms has become essential given the multifactorial nature of many common health conditions. Examples include the identification of hub genes associated with the etiology of intervertebral disc (IVD) (912). Inflammatory cytokines play a significant role in disc degeneration (13). As disc degeneration progresses, the expression of proinflammatory cytokines, such as interleukin-1 $\beta$ (IL-1 $\beta$ ) and tumor necrosis factor alpha (TNF- $\alpha$ ) in the nucleus pulposus (NP) tissue increase significantly, which could accelerate disc cell degeneration by promoting the release of matrix metalloproteinases (MMPs) and inflammatory cytokines (13-16). However, Le et al. believed that IL-1 may have a more significant role than TNF- $a$ (16). Furthermore, IL-1 $\beta$ has been shown to elevate both neurotrophin gene expression levels and the production of nerve growth factor (NGF), which is associated with low back pain (17). Thereafter, inflammatory processes exacerbated by IL-1 $\beta$ are believed to be key mediators of disc degeneration and low back pain, and IL-1 $\beta$ may be a better target for therapeutic intervention of IVDD.

In this study, microarray data profiling of human disc tissue samples from patients with herniated discs and degenerative disc disease, including 4 samples stimulated with $10^{2} \mathrm{pM} \mathrm{IL-1} \beta$ and 4 controls performed by Gruber et al. (GSE27494) were downloaded and reanalyzed. Differentially expressed genes (DEGs) were identified and co-expression network analysis was performed, followed by

Page $2 / 21$ 
functional analysis of the genes in the network. DEGs were subsequently subjected to functional and pathway annotations, construction of protein-protein interaction (PPI) networks, and module analysis, to investigate the critical DEGs in the progression of IDD. The present study aimed to identify the molecular markers indicating the induction of disc degeneration by inflammatory cytokines, which may guide further investigations and contribute to the development of novel therapeutic strategies for the treatment of IDD.

\section{Materials And Methods}

\section{Collection of microarray data}

The gene profile GSE27494, which was downloaded from the Gene Expression Omnibus (GEO) database (https://www.ncbi.nlm.nih.gov/geo/), was produced by the [U133_X3P] Affymetrix Human X3P Array (Platform GPL1352). The gene profile consisted of four degenerated disc tissue samples and four degenerative disc tissues subjected to additional IL-1 $\beta$ exposure. Disc tissue samples were obtained from surgical disc procedures performed on patients with herniated discs and degenerative disc disease. Cultured annulus cells were grown in a 3D collagen construct with or without $10^{2} \mathrm{pM} \mathrm{IL}-1$ for a total of 14 days. Following homogenization in TRIzol reagent, the total RNA was isolated and analyzed using microarrays.

\section{Identification of differentially expressed genes (DEGs)}

The limma R package emerged as one of the most widely used statistical tests for identifying DEGs (18). The package was obtained from the open website (http://www.bioconductor.org/packages/release/bioc/html/limma.html). We screened DEGs between IL-1 $\beta$ exposed and control disc cells by utilizing the limma package with an adjusted P-value $<0.05$ and a log (fold change) $>2$ or log (fold change) $<-2$ as the cut-off criteria. Furthermore, volcano plots and heat maps were drawn using $\mathrm{R}$ software.

\section{Functional Annotation of DEGs using a database for annotation, visualization and integrated discovery (DAVID), and Metascape analysis}

We applied DAVID (https://david.ncifcrf.gov/home.jsp) (version 6.8) (19) to perform Gene Ontology (GO) functional analysis (20) and performed Kyoto Encyclopedia of Genes and Genomes (KEGG) pathway analysis of DEGs (21). The enriched GO terms and pathways with a P-value $<0.05$ were identified. Moreover, GO and KEGG pathway analyses were performed using Metascape (http://metascape.org/gp/index.html\#/main/step1) (22). Terms with a p-value $<0.01$, a minimum count of 3 , and an enrichment factor $>$ 1.5 (enrichment factor is the ratio between the observed count and the count expected by chance) were collected and grouped into clusters based on their membership similarities.

\section{Enrichment analysis of GO and KEGG using gene set enrichment analysis (GSEA)}

GSEA primarily analyzes microarray data, using genomic and genetic sequencing to detect the significant biological differences in microarray datasets (23). In the present study, we performed GESA analysis on the gene sequences of IL-1 $\beta$-exposed and control disc cells using c5.all.v7.1.symbols.gmt, and c2.cp.kegg.v7.1.symbols.gmt as reference gene sets (software.broadinstitute.org/gsea/) (24). Gene set permutations were analyzed 1,000 times. In addition, the normalized enrichment score, normalized P-value, and false discovery rate (FDR) q values were applied to filter the correlative $\mathrm{GO}$ terms and pathways.

\section{Construction of the protein-protein interaction (PPI) network, significant module, and hub gene network}

First, we used Metascape (http://metascape.org/gp/index.html\#/main/step1) (22) to construct the PPI network and screen the significant module. Second, to assess the functional associations among the target genes of upregulated and downregulated DEGs, we uploaded the target gene data to the Search Tool for the Retrieval of Interacting Genes (STRING, http://string.embl.de/) to construct the PPI network. Interactions with a combined score $>0.7$ were considered significant. Highly interconnected (hub) genes in the PPI network were analyzed using Cytoscape software (version 3.6.0). The Molecular Complex Detection tool (MCODE) (version 1.5.1) (25) could screen and identify the most significant module in the PPI network. Genes with the top 10 highest degrees in the PPI network were viewed as hub genes.

\section{Identification of significant genes}

We delineated a Venn diagram to identify the significant common genes among "Metascape_MCODE", "Cytoscape_MCODE", and "Cytoscape_cytoHubba" using Fun Rich software (http://www.funrich.org) (25). Summaries of the function of significant genes were obtained via DAVID (https://david.ncifcrf.gov/home.jsp). The R language was used to perform the clustering analysis of significant genes based on the gene expression levels. 


\section{Results}

\section{Screening for DEGs}

After the analysis of the datasets (GSE27494) using the limma package, the DEGs between IL-1 $\beta$-exposed and control disc cells could be presented in a volcano plot (Fig. 1). After setting the threshold value, a total of $260 \mathrm{DEGs}$ were reserved, including 161 upregulated DEGs (Table 1) and 99 down-regulated DEGs (Table 2). The cluster heatmap of these DEGs is shown in Fig. 2.

Table 1 The genes that were up-regulated by a fold-change $>2$ and with a $p<0.05$ (top 20).

\begin{tabular}{|lllll|}
\hline Gene symbol & Ensembl gene ID & Log FC & P-value & adj.P.Val \\
\hline CXCL5 & ENSG00000163735 & 9.83 & $9.95 \times 10-06$ & 0.017479 \\
\hline CXCL8 & ENSG00000169429 & 7.88 & $4.72 \times 10-06$ & 0.012986 \\
\hline IL1B & ENSG00000125538 & 7.29 & $3.10 \times 10-05$ & 0.027488 \\
\hline MMP3 & ENSG00000149968 & 7.18 & $1.68 \times 10-04$ & 0.057873 \\
\hline SLC7A2 & ENSG00000003989 & 6.90 & $4.16 \times 10-06$ & 0.012986 \\
\hline C15orf48 & ENSG00000166920 & 6.27 & $1.41 \times 10-05$ & 0.021941 \\
\hline CXCL3 & ENSG00000163734 & 6.24 & $1.02 \times 10-05$ & 0.017479 \\
\hline CCL3L3 & ENSG00000276085 & 6.20 & $1.04 \times 10-03$ & 0.120405 \\
\hline CXCL2 & ENSG00000081041 & 6.18 & $1.62 \times 10-05$ & 0.022413 \\
\hline IL11 & ENSG00000095752 & 6.10 & $5.88 \times 10-05$ & 0.036427 \\
\hline IL6 & ENSG00000136244 & 5.87 & $2.27 \times 10-03$ & 0.169458 \\
\hline HSD11B1 & ENSG00000117594 & 5.49 & $4.31 \times 10-05$ & 0.03215 \\
\hline LIF & ENSG00000128342 & 5.35 & $8.59 \times 10-06$ & 0.016999 \\
\hline RSP03 & ENSG00000146374 & 5.35 & $2.46 \times 10-05$ & 0.027393 \\
\hline MMP8 & ENSG00000118113 & 5.29 & $4.37 \times 10-06$ & 0.012986 \\
\hline CLDN1 & ENSG00000163347 & 5.17 & $9.33 \times 10-06$ & 0.017479 \\
\hline ERC2 & ENSG00000187672 & 5.11 & $3.09 \times 10-05$ & 0.027488 \\
\hline EHF & ENSG00000135373 & 5.02 & $2.05 \times 10-04$ & 0.063472 \\
\hline BMP2 & ENSG00000125845 & 4.89 & $6.30 \times 10-04$ & 0.098134 \\
\hline RAB27B & ENSG00000041353 & 4.86 & $7.02 \times 10-06$ & 0.01485 \\
\hline
\end{tabular}

FC, fold change.

Table 2 The genes that were down-regulated by a fold-change $<-2$ and with a $p<0.05$ (top 20 ). 


\begin{tabular}{|lllll|}
\hline Gene symbol & Ensembl gene ID & Log FC & P-value & adj.P.Val \\
\hline FGL2 & ENSG00000127951 & -5.42 & $9.61 \times 10-05$ & 0.047186 \\
\hline LSP1 & ENSG00000130592 & -5.03 & $6.50 \times 10-04$ & 0.100144 \\
\hline ACTC1 & ENSG00000159251 & -4.94 & $2.86 \times 10-02$ & 0.48192 \\
\hline FAM107A & ENSG00000168309 & -4.75 & $9.74 \times 10-03$ & 0.307323 \\
\hline MAP1LC3C & ENSG00000197769 & -4.67 & $4.10 \times 10-04$ & 0.080189 \\
\hline CRIP1 & ENSG00000213145 & -4.59 & $7.58 \times 10-04$ & 0.107444 \\
\hline EXOSC7 & ENSG00000075914 & -4.49 & $2.16 \times 10-05$ & 0.025494 \\
\hline COL15A1 & ENSG00000204291 & -4.34 & $7.55 \times 10-03$ & 0.275959 \\
\hline COMP & ENSG00000105664 & -4.29 & $4.53 \times 10-02$ & 0.573519 \\
\hline ASPN & ENSG00000106819 & -4.10 & $1.03 \times 10-02$ & 0.316056 \\
\hline ADIRF & ENSG00000148671 & -4.03 & $1.27 \times 10-03$ & 0.132417 \\
\hline OGN & ENSG00000106809 & -4.00 & $1.51 \times 10-03$ & 0.14488 \\
\hline SMOC2 & ENSG00000112562 & -3.96 & $2.70 \times 10-04$ & 0.070341 \\
\hline OLFML2B & ENSG00000162745 & -3.79 & $1.75 \times 10-04$ & 0.058275 \\
\hline PDE5A & ENSG00000138735 & -3.76 & $3.24 \times 10-04$ & 0.072076 \\
\hline SLC14A1 & ENSG00000141469, & -3.63 & $4.25 \times 10-02$ & 0.557619 \\
\hline SNED1 & ENSG00000162804 & -3.56 & $1.72 \times 10-03$ & 0.152379 \\
\hline KCNN4 & ENSG00000104783 & -3.35 & $2.59 \times 10-03$ & 0.176067 \\
\hline TOX & ENSG00000198846 & -3.34 & $5.00 \times 10-03$ & 0.23011 \\
\hline ANK3 & ENSG00000151150 & -3.30 & $2.19 \times 10-03$ & 0.167475 \\
\hline
\end{tabular}

FC, fold change.

\section{The enrichment results of GO and KEGG via DAVID and Metascape}

The GO functional annotation analysis of the DEGs showed that (1) the biological processes were mainly involved in the regulation of chemotaxis, taxis, and the response to molecules of bacterial origin; (2) the molecular functions of the altered genes were mainly related to cytokine activity, cytokine receptor binding, and chemokine receptor binding; and (3) the cell components involved were mainly in the extracellular region and extracellular matrix (Fig. 3A-C). In the KEGG signaling pathway analysis, the 260 DEGs were found to be mainly involved in the interactions of cytokine-cytokine receptor interaction, rheumatoid arthritis, TNF signaling pathway, salmonella infection, chemokine signaling pathway, and other signal transduction pathways (Fig. 3D).

Furthermore, the functional enrichment analysis performed using Metascape showed that the DEGs were significantly enriched in NABA MATRISOME ASSOCIATED, extracellular matrix organization, NABA CORE MATRISOME, positive regulation of cell motility, and regulation of cell adhesion (Fig. 4A-C).

\section{GO and KEGG pathway enrichment analysis of DEGs using GSEA}

The results of GO enrichment analysis using GSEA indicated that 3,186/5,154 genes were upregulated in IL-1 1 -exposed disc cells. The most significant enriched genes in the upregulated and downregulated gene sets according to the normalized enrichment score are orderly listed in Table 3. Fig. 5 shows the six most significant plots in the up-regulated and down-regulated gene sets. KEGG enrichment analysis using GSEA indicated that 107/177 gene sets were upregulated in IL-1 $\beta$-exposed disc cells compared to control disc cells, while 70/177 gene sets were down-regulated. The most significant enriched genes in the up-regulated and down-regulated gene sets according to the normalized enrichment score are orderly listed in Table 4. Fig. 6 shows the six most significant plots in the up-regulated and downregulated gene sets. 
Table 3 Functional enrichment analysis of DEGs in IL-1 $\beta$-exposed disc cells using GSEA.

\begin{tabular}{|c|c|c|c|c|}
\hline Gene Set Name & SIZE & ES & NES & $\begin{array}{l}\text { P- } \\
\text { value }\end{array}$ \\
\hline \multicolumn{5}{|l|}{ Upregulated } \\
\hline GO_NEUTROPHIL_MIGRATION & 103 & 0.835 & 2.461 & 0.000 \\
\hline GO_ANTIMICROBIAL_HUMORAL_IMMUNE_RESPONSE_MEDIATED_BY_ANTIMICROBIAL_PEPTIDE & 58 & 0.895 & 2.387 & 0.000 \\
\hline GO_GRANULOCYTE_MIGRATION & 125 & 0.789 & 2.387 & 0.000 \\
\hline GO_CYTOKINE_ACTIVITY & 194 & 0.750 & 2.366 & 0.000 \\
\hline GO_CHEMOKINE_RECEPTOR_BINDING & 50 & 0.891 & 2.354 & 0.000 \\
\hline GO_CHEMOKINE_ACTIVITY & 41 & 0.908 & 2.335 & 0.000 \\
\hline \multicolumn{5}{|l|}{ Downregulated } \\
\hline GO_REGULATION_OF_INOSITOL_PHOSPHATE_BIOSYNTHETIC_PROCESS & 16 & 0.842 & 1.810 & 0.004 \\
\hline GO_IMMUNOGLOBULIN_RECEPTOR_BINDING & 15 & 0.852 & 1.795 & 0.000 \\
\hline GO_WATER_TRANSPORT & 16 & 0.821 & 1.753 & 0.000 \\
\hline GO_CEREBRAL_CORTEX_NEURON_DIFFERENTIATION & 24 & 0.763 & 1.735 & 0.002 \\
\hline GO_EXTRACELLULAR_MATRIX_STRUCTURAL_CONSTITUENT & 157 & 0.558 & 1.731 & 0.000 \\
\hline GO_REGULATION_OF_MEMBRANE_DEPOLARIZATION & 43 & 0.676 & 1.728 & 0.000 \\
\hline
\end{tabular}

ES: Enrichment Score; NES: Normalized Enrichment Score.

Table 4 Pathway enrichment analysis of DEGs using GSEA

\begin{tabular}{|lllll|}
\hline Gene Set Name & SIZE & ES & NES & P-value \\
\hline Upregulated & & & & \\
\hline KEGG_CYTOKINE_CYTOKINE_RECEPTOR_INTERACTION & 243 & 0.742 & 2.420 & 0.000 \\
\hline KEGG_CHEMOKINE_SIGNALING_PATHWAY & 171 & 0.665 & 2.075 & 0.000 \\
\hline KEGG_GRAFT_VERSUS_HOST_DISEASE & 35 & 0.823 & 2.035 & 0.000 \\
\hline KEGG_NOD_LIKE_RECEPTOR_SIGNALING_PATHWAY & 62 & 0.740 & 2.002 & 0.000 \\
\hline KEGG_TOLL_LIKE_RECEPTOR_SIGNALING_PATHWAY & 96 & 0.654 & 1.931 & 0.000 \\
\hline KEGG_HEMATOPOIETIC_CELL_LINEAGE & 75 & 0.678 & 1.900 & 0.000 \\
\hline DOWnregulated & & & & \\
\hline KEGG_BASE_EXCISION_REPAIR & 33 & 0.617 & 1.493 & 0.033 \\
\hline KEGG_THYROID_CANCER & 29 & 0.607 & 1.475 & 0.045 \\
\hline KEGG_DILATED_CARDIOMYOPATHY & 85 & 0.498 & 1.422 & 0.021 \\
\hline KEGG_DNA_REPLICATION & 36 & 0.536 & 1.351 & 0.069 \\
\hline KEGG_PEROXISOME & 75 & 0.438 & 1.243 & 0.112 \\
\hline KEGG_TGF_BETA_SIGNALING_PATHWAY & 84 & 0.423 & 1.219 & 0.113 \\
\hline
\end{tabular}

ES: Enrichment Score; NES: Normalized Enrichment Score

PPI construction and module analysis

Page 6/21 
Using Metascape analysis, a PPI network of DEGs was constructed (Fig. 7A). Six MCODE modules were identified from the PPI network. First, MCODE 1 consisted of 12 genes, including CXCL2, CCL20, SAA1, GPER1, CXCL8, CXCL1, C3, ACKR3, PF4, CXCL5, CXCL3, and CXCL6 (Fig. 7B). Second, MCODE 2 consisted of 5 genes, including ACTC1, GCH1, TUBB3, ACTG2, and SOD2 (Fig. 7C). Third, MCODE 3 consisted of 3 genes, TF, TFRC, and AMPH (Fig. 7D). Fourth, MCODE 4 consists of 3 genes: Lyn, IL6, and IL1B (Fig. 7E). Fifth, MCODE 5 consists of 3 genes: MMP3, CCL7, and CCL2 (Fig. 7F). Sixth, MCODE 6 consists of 3 genes: CCKAR, GNA14, and P2RY6 (Fig. 7 G).

Furthermore, the PPI relationship networks of the DEGs were also generated using the STRING online tool, and there were 327 edges and 251 nodes in the PPI network (PPI enrichment p-value < 0.05) (Fig. 8A). Six MCODE modules were also identified from the PPI network using the MCODE (version 1.5.1), one plug-in of Cytoscape (Fig. 8B-G). A total of 10 genes were identified as hub genes using Cytoscape: CXCL8, CXCL1, CCL20, CXCL2, CXCL5, CXCL3, CXCL6, C3, PF4, and GPER1 (Fig. 8H).

\section{Identification and analysis of significant genes}

The VENN diagram showed that there were ten significant common genes among "Metascape_MCODE," "Cytoscape_MCODE," and "Cytoscape_cytoHubba,": CXCL8, CXCL1, CCL20, CXCL2, CXCL5, CXCL3, CXCL6, C3, PF4, and GPER1 (Fig. 9A). Hierarchical clustering showed that the significant genes could be used to significantly distinguish IL-1 $\beta$-exposed disc cells from control cells. Compared with the control cells, the expression of GPER1 was downregulated, and the expression of CXCL8, CXCL1, CCL20, CXCL2, CXCL5, CXCL3, CXCL6, C3, and PF4 was upregulated in IL-1ß-exposed discs (Fig. 9B).

\section{Discussion}

Biological information analysis has been widely applied to help scientists identify new therapeutic targets by exploring gene changes in the process of disease development $(26,27)$. In the present study, we focused on inflammation as the primary etiological factor impacting disc degeneration, and IL-1 $\beta$ was selected as a representative inflammatory cytokine (28). Therefore, we obtained gene expression profiling data from GEO microarray datasets containing 4 samples of degenerative intervertebral disc cells stimulated with $10^{2} \mathrm{pM} \mathrm{IL-1} \beta$ and 4 controls. The key genes that were affected during disc degeneration caused by inflammatory cytokine exposure were analyzed using bioinformatics analysis, and 260 DEGs were obtained (161 upregulated and 99 downregulated). Subsequently, GO annotation and KEGG pathway enrichment analyses of the DEGs revealed their participation in several cellular processes (e.g., the regulation of chemotaxis and taxis) and signaling cascades related to IVDD development (e. g., the interactions of cytokine-cytokine receptor interaction and rheumatoid arthritis). A PPI network based on these genes was constructed to obtain the top 10 hub genes: CXCL8, CXCL1, CCL20, CXCL2, CXCL5, CXCL3, CXCL6, C3, PF4, and GPER1. Subsequent analysis confirmed their involvement in important IVDD-related pathways. Our results suggest that these hub genes might be potential biological targets in IVD diagnosis and for the development of therapeutic drugs.

In terms of GO enrichment analysis, we found that most of DEGs were mainly involved in the regulation of chemotaxis and taxis, cytokine activity, cytokine receptor binding, and chemokine receptor binding. Regarding the KEGG pathway, we similarly found that most of DEGs were primarily enriched in the cytokine-cytokine receptor interaction, TNF signaling pathway, chemokine signaling pathway. In addition, CXCL5 and CXCL8 are the most significantly upregulated genes in our analysis. Inflammation has been correlated with IDD but its role remains controversial. Previous studies have shown that pathological inflammation of IVD and peridiscal space is characterized by increased levels of pro-inflammatory cytokines and chemokines (29-31). Cytokines and chemokines have three modes of action: (1) recruiting inflammatory cells and activating phagocytosis, (2) stimulating the production of other inflammatory mediators and MMPs, and (3) enhancing matrix degradation (32-37). And these effects can activate a cascade of detrimental self-promoting events that exacerbate the degeneration of IVD. Furthermore, experimental studies have reported the contribution of pro-inflammatory cytokines in degenerated painful IVD $(38,39)$.

In addition, our study also showed that the DEGs were mostly enriched in the extracellular region and extracellular matrix. A normal adult IVD is composed of a vascular tissue, which contain a large amount of extracellular matrix (ECM), and the disc cells are responsible for maintaining the integrity of the ECM (40). Changes in the metabolic balance of IVD cells affect the quality and quantity of the ECM and its functional properties, and these changes can, therefore, be related to disc degeneration (41). The underlying molecular mechanisms of IVDD have been previously investigated, and ECM degradation and inflammation have been confirmed to play a critical role in accelerating IVDD progression. Le et al. reported that IL-1 $\beta$ induced the expression of MMPs (16). And the study of Vo et al. found that upregulation of MMP and ADAMTS expression and enzymatic activity is implicated in disc ECM destruction, leading to the development of IDD (42). Our bioinformatics analysis also showed that MMP-3 significantly increased in the degenerative intervertebral disc cells stimulated with $10^{2} \mathrm{pM} \mathrm{IL-1 \beta}$ than controls. 
Furthermore, we constructed a PPI network the top 10 hub genes - CXCL8, CXCL1, CCL20, CXCL2, CXCL5, CXCL3, CXCL6, C3, PF4, and GPER1 - were identified. It is known that leukocytes, such as neutrophils, macrophages, and lymphocytes, mainly participate in the inflammatory defense response of the body. Chemokines produced by mammalian cells during inflammation are a class of chemotactic and inducible small molecule peptides that are ubiquitous and play an important role in acute and chronic inflammation (43). Based on key cysteine residues involved in disulfide bonds, chemokines are classified as C, CC, CXC, and CX3C (44). Among the top 10 hub genes, CXCL8, CXCL1, CXCL2, CXCL5, CXCL3, CXCL6, and PF4 belong to the CXC family of chemokines, CCL20 belongs to the CC family, and C3 belongs to the $\mathrm{C}$ family.

CXCL8 is a chemotactic factor that attracts neutrophils, basophils, and T-cells. It is involved in neutrophil activation and modulates both acute and chronic inflammation. Ahn et al. found that CXCL8 mRNA expression was associated with the development of radicular pain by back extension and suggested that CXCL8 participates in the pathomechanism of nerve root inflammation in lumbar disc herniations (45). Pederson et al. found that patients with lumbar radicular pain due to disc herniation have increased serum levels of CXCL8 (46). Wang et al. also found higher CXCL8 concentrations in the serum of lumbar disc herniation (LDH) patients (47). A recent study reported that CXCL8 was elevated in the cerebrospinal fluid (CSF) of chronic low back pain (LBP) patients with IVDD, compared to pain-free subjects with or without IVDD, and they supported that the IL-8 signaling pathway is a viable therapy for chronic LBP (48). Palada et al. considered that neuroinflammation mediated by elevated CXCL8 concentrations in the CSF and CXCL8 mediated periphery-to-CNS (central nervous system) inflammatory cross-talk contributes to pain in LDH patients (49). Burke et al. reported that the increased level of CXCL8 within the nucleus pulposus may be related to neovascularization in patients with discogenic pain (50). Importantly, the protein expression of CXCL8 is significantly increased, concordant with the histological degenerative tissue changes in human NP (51). Similarly, Zhang et al. found that annulus fibrosus samples from LBP patients had an elevated IL-8 expression compared to controls with scoliosis (52). These experimental results are consistent with the results of our analysis. In the present study, the expression of CXCL8 in IL-1 $\beta$ exposed samples was increased by 7.88 -fold compared to control samples, indicating that increased levels of IL-1 $\beta$ affected the expression of the low-back-pain-related factors in intervertebral disc cells, which might become a new target for treating related diseases.

Chemokine CXCL5, which was increased by 9.83-fold in the present study, also called epithelial neutrophil-activating peptide 78 (ENA-78), belongs to the CXC family of chemokines that carry a glutamate-leucine-arginine (ELR) motif and binds to the G-protein CXCR2 to recruit neutrophils and promote angiogenesis $(53,54)$. CXCL5 has been shown to facilitate nociceptive input transmission in the pathogenesis of inflammatory pain (55). Dawes et al. reported that the chemokine CXCL5 is a peripheral mediator of ultraviolet B (UVB)-induced inflammatory pain, likely in humans and rats (56). Xu et al. reported that the upregulation of spinal CXCL5 and CXCR2 is involved in neuropathic pain after nerve injury by regulating GSK-3 $\beta$ activity in a rat model of chronic constriction injury (CCI) of the sciatic nerves (57). In addition, CXCL5 overexpression has been observed in several malignancies, such as pancreatic ductal adenocarcinoma, demonstrating its role in tumor carcinogenesis (58). This finding showed that the role of CXCL5 in intervertebral discs merits further research.

Chemokine CXCL6, also known as granulocyte chemotactic protein 2 (GCP2), recruits inflammatory cells to the site of inflammation by binding to receptors CXCR1 and CXCR2. Sandell et al. reported that the expression of CXCL6 was significantly increased in IL-1 $\beta$-treated human chondrocytes (59). CXCL6 expression in IL-1 $\beta$-exposed samples was increased by 3.2-fold, which was consistent with the results of a previous study. CXCL6 has also been detected in conditioned medium of induced degenerative discs in organ culture and may contribute to the chemotactic response of induced-degenerative discs (60). Grad et al. reported that significantly elevated levels of systemic blood plasma concentrations of chemokine CXCL6 were observed in individuals with moderate/severe disc degeneration, in comparison with a control group with no signs of disc degeneration, according to the MRI results (61). However, long-term medically treated patients with persistent chronic back pain expressed lower levels of the chemokine CXCL6 compared to pain-free healthy controls (62).

CXCL1 promotes both nociceptor and central sensitization through its primary receptor CXCR2, which may be a promising target for novel analgesic drugs in pathological pain conditions triggered by either peripheral inflammation or nerve injury (63). Under varied pathological states, activated astrocytes are considered the main source of CXCL1 (64). In addition, previous studies indicated that CXCL1 might act on CXCR2 via glial-neuronal interactions in the spinal cord in several pathological pain models $(65,66)$. A study by Ni et al. also demonstrated that CXCL1-CXCR2 signaling plays a critical role in glial-neuron interactions and in descending facilitation of bone cancer pain (BCP) (67). In our study, CXCL1 expression was increased 3.97-fold, indicating that CXCL1 plays an important role in LBP with IVDD.

CC chemokine ligand 20 (CCL20), which is expressed by endothelial cells in several tissues, is also known as macrophage inflammatory protein 3a (MIP-3a); it is a 70-amino-acid chemokine that binds exclusively to the chemokine receptor 6 (CCR6) and recruits CCR6- 
expressing cells $(68,69)$. Zhang et al. reported that NP cells from the extruded and herniated patient group could produce abundant amounts of CCL20 (70). Subsequently, Zhang et al. suggested a potential mechanism for the recruitment of IL-17-producing cells to degenerated intervertebral discs via a CCL20/CCR6 system in vivo, while IL-17 is involved in the auto-immune process of intervertebral disc degeneration in rat models (71).

G protein-coupled estrogen receptor 1 (GPER1) is the receptor of estrogen. There are four majors naturally occurring oestrogens identified in women: oestrone (E1), oestradiol (E2), oestriol (E3), and estetrol (E4). In addition, oestrogen receptors consist of two classic nuclear receptors, estrogen receptor (ER)- $\alpha$ and ER- $\beta$, and the membrane-bound G-protein-coupled receptor 30 (GPR30) (72). Over the past decade, many researchers have widely studied the relevance between oestrogen and IVDD and found that oestrogen can effectively alleviate IVDD development by inhibiting the apoptosis of IVD cells. Oestrogen can decrease IVD cell apoptosis in multiple ways, including the inhibition of inflammatory cytokines IL-1 $\beta$ and TNF-a, reducing catabolism because of matrix metalloproteinases inhibition, upregulating integrin a $2 \beta 1$ and IVD anabolism, activating the PI3K/Akt pathway, decreasing the oxidative damage, and promoting autophagy (72). Song et al. found that both cytoplasmic and nuclear staining of ERa and ER $\beta$ immunoreactivity were observed in nucleus pulposus cells, and ERa and ER $\beta$ expression significantly decreased, along with the aggravation of IVD degeneration in both males and females (73). Wei et al. also showed that GPR30 with a high affinity for estrogen is expressed in the human disc NP, can mediate E2 enhanced cell proliferation and influence disc cell survival (74). In our study, GPER1 was downregulated by 2.42 -fold. Therefore, IL-1 $\beta$ decreases the expression of GPER1 in human disc NP, which accelerates disc degeneration.

Chemokine (C-X-C motif) ligand 2 (CXCL2), also known as macrophage inflammatory protein 2 (MIP-2), belongs to the CXC chemokine family, along with growth-regulated protein $\beta$ and growth-regulated oncogene-2. CXCL2 is $90 \%$ identical regarding the amino acid sequence to the related chemokine CXCL1. CXCL2, which is produced in an injured sciatic nerve by partial ligation and is secreted by monocytes, can rapidly recruit neutrophils to sites of inflammation or injured tissues through blood flow, in response to infection or injury $(43,75,76)$. CXCL3 is a small cytokine belonging to the CXC chemokine family, and is also known as the GRO3 oncogene, GRO protein gamma, and macrophage inflammatory protein $2 \beta$. PF4, also known as CXCL4, which belongs to the CXCL chemokine family, is predominantly produced by megakaryocytes and a-granules of platelets and has an important role in hemostasis/thrombosis (77). Complement C3 is a central molecule in the complement system and plays a very important role in inflammatory and immune responses. Interestingly, the complement system has been implicated in cartilage degradation, and C3 has been found to be aberrantly increased in the synovial fluids from individuals with osteoarthritis and in animal models of osteoarthritis $(78,79)$. However, we did not retrieve the literature to identify whether IVDD is correlated with CXCL2, CXCL3, C3, and PF4.

\section{Conclusions}

In this study, bioinformatic analysis of the gene expression profiles of degenerative intervertebral disc cells stimulated with IL-1 $1 \beta$ showed that CXCL8 and other 9 key genes may play a role in the development of disc degeneration induced by inflammatory reactions. This suggests that bioinformatics methods can be used to identify potential therapeutic target genes and provide new insights into intervertebral disc degeneration.

\section{Abbreviations}

DEGs: Differentially expressed genes; DAVID: Database for Annotation, Visualization, and Integrated Discovery; GSEA: Gene Set Enrichment Analysis; STRING: Search Tool for the Retrieval of Interacting Genes; GO: Gene Ontology; KEGG: Kyoto Encyclopedia of Genes and Genomes; TNF: Tumor necrosis factor; IVDD: Intervertebral disc degeneration; IVD: Intervertebral disc; IL-1 $\beta$ : Interleukin-1 $\beta$; NP: Nucleus pulposus; MMPs: Matrix metalloproteinases; NGF: Nerve growth factor; PPI: Protein-protein interaction; GEO: Gene Expression Omnibus; FDR: False discovery rate; MCODE: Molecular Complex Detection tool; FC: Fold change; ES: Enrichment score; NES: Normalized enrichment score; ECM: Extracellular matrix; LDH: Lumbar disc herniation; CSF: Cerebrospinal fluid; LBP: Low back pain; ELR: Glutamateleucine-arginine; UVB: Ultraviolet B; CCl: Chronic constriction injury; GCP2: Granulocyte chemotactic protein 2; BCP: Bone cancer pain; CCL20: CC chemokine ligand 20; MIP-3a: Macrophage inflammatory protein 3a; CCR6: Chemokine receptor 6; GPER1: G protein-coupled estrogen receptor 1; ER: Estrogen receptor; GPR30: G-protein-coupled receptor 30; CXCL2: Chemokine (C-X-C motif) ligand 2; MIP-2: Macrophage inflammatory protein 2

\section{Declarations}

\section{Ethics approval and consent to participate}


Not applicable.

\section{Consent for publication}

Not applicable.

\section{Availability of data and materials}

The datasets used and/or analysed during the current study are available from the corresponding author on reasonable request.

\section{Competing interests}

The authors declare that they have no competing interests.

\section{Funding}

Our study was supported by the project of medical discipline priority of Shijingshan District, Beijing.

\section{Authors' contributions}

NF and SY: design of the study and writing of the manuscript. YH, PD, JL, XK, WZ, YL and LZ: design of the study and revising manuscript critically for important intellectual content. All authors have read and approved the final manuscript.

\section{Acknowledgements}

Not applicable.

\section{References}

1. Vergroesen PP, Kingma I, Emanuel KS, Hoogendoorn RJ, Welting TJ, van Royen BJ, et al. Mechanics and biology in intervertebral disc degeneration: a vicious circle. Osteoarthritis Cartilage. 2015;23(7):1057-70.

2. Liao Z, Wu X, Song Y, Luo R, Yin H, Zhan S, et al. Angiopoietin-like protein 8 expression and association with extracellular matrix metabolism and inflammation during intervertebral disc degeneration. Journal of cellular and molecular medicine. 2019;23(8):573750.

3. Teraguchi M, Yoshimura N, Hashizume H, Yamada H, Oka H, Minamide A, et al. Progression, incidence, and risk factors for intervertebral disc degeneration in a longitudinal population-based cohort: the Wakayama Spine Study. Osteoarthritis Cartilage. 2017;25(7):1122-31.

4. Dario AB, Ferreira ML, Refshauge KM, Lima TS, Ordoñana JR, Ferreira PH. The relationship between obesity, low back pain, and lumbar disc degeneration when genetics and the environment are considered: a systematic review of twin studies. The spine journal : official journal of the North American Spine Society. 2015;15(5):1106-17.

5. Sivan SS, Wachtel E, Roughley P. Structure, function, aging and turnover of aggrecan in the intervertebral disc. Biochimica et biophysica acta. 2014;1840(10):3181-9.

6. Hangai M, Kaneoka K, Kuno S, Hinotsu S, Sakane M, Mamizuka N, et al. Factors associated with lumbar intervertebral disc degeneration in the elderly. The spine journal : official journal of the North American Spine Society. 2008;8(5):732-40.

7. Wang YXJ. Postmenopausal Chinese women show accelerated lumbar disc degeneration compared with Chinese men. Journal of orthopaedic translation. 2015;3(4):205-11.

8. Zhao L, Manchikanti L, Kaye AD, Abd-Elsayed A. Treatment of Discogenic Low Back Pain: Current Treatment Strategies and Future Options-a Literature Review. Current pain and headache reports. 2019;23(11):86.

9. Sampara P, Banala RR, Vemuri SK, Av GR, Gpv S. Understanding the molecular biology of intervertebral disc degeneration and potential gene therapy strategies for regeneration: a review. Gene therapy. 2018;25(2):67-82.

10. Zhang Y, He F, Chen Z, Su Q, Yan M, Zhang Q, et al. Melatonin modulates IL-1 $\beta$-induced extracellular matrix remodeling in human nucleus pulposus cells and attenuates rat intervertebral disc degeneration and inflammation. Aging. 2019;11(22):10499-512.

11. Wang K, Chen T, Ying X, Zhang Z, Shao Z, Lin J, et al. Ligustilide alleviated IL-1 $\beta$ induced apoptosis and extracellular matrix degradation of nucleus pulposus cells and attenuates intervertebral disc degeneration in vivo. International immunopharmacology. 2019;69:398-407. 
12. Gorth DJ, Shapiro IM, Risbud MV. A New Understanding of the Role of IL-1 in Age-Related Intervertebral Disc Degeneration in a Murine Model. Journal of bone and mineral research : the official journal of the American Society for Bone and Mineral Research. 2019;34(8):1531-42.

13. Risbud MV, Shapiro IM. Role of cytokines in intervertebral disc degeneration: pain and disc content. Nature reviews Rheumatology. 2014;10(1):44-56.

14. Tu J, Li W, Zhang Y, Wu X, Song Y, Kang L, et al. Simvastatin Inhibits IL-1 $\beta$-Induced Apoptosis and Extracellular Matrix Degradation by Suppressing the NF-kB and MAPK Pathways in Nucleus Pulposus Cells. Inflammation. 2017;40(3):725-34.

15. Huang KY, Hsu YH, Chen WY, Tsai HL, Yan JJ, Wang JD, et al. The roles of IL-19 and IL-20 in the inflammation of degenerative lumbar spondylolisthesis. Journal of inflammation (London, England). 2018;15:19.

16. Le Maitre CL, Hoyland JA, Freemont AJ. Catabolic cytokine expression in degenerate and herniated human intervertebral discs: IL1 beta and TNFalpha expression profile. Arthritis research \& therapy. 2007;9(4):R77.

17. Gruber HE, Hoelscher GL, Bethea S, Hanley EN, Jr. Interleukin 1-beta upregulates brain-derived neurotrophic factor, neurotrophin 3 and neuropilin 2 gene expression and NGF production in annulus cells. Biotechnic \& histochemistry : official publication of the Biological Stain Commission. 2012;87(8):506-11.

18. Ritchie ME, Phipson B, Wu D, Hu Y, Law CW, Shi W, et al. limma powers differential expression analyses for RNA-sequencing and microarray studies. Nucleic acids research. 2015;43(7):e47.

19. Huang DW, Sherman BT, Tan Q, Collins JR, Alvord WG, Roayaei J, et al. The DAVID Gene Functional Classification Tool: a novel biological module-centric algorithm to functionally analyze large gene lists. Genome biology. 2007;8(9):R183.

20. Mootha VK, Lindgren CM, Eriksson KF, Subramanian A, Sihag S, Lehar J, et al. PGC-1alpha-responsive genes involved in oxidative phosphorylation are coordinately downregulated in human diabetes. Nature genetics. 2003;34(3):267-73.

21. Szklarczyk D, Franceschini A, Wyder S, Forslund K, Heller D, Huerta-Cepas J, et al. STRING v10: protein-protein interaction networks, integrated over the tree of life. Nucleic acids research. 2015;43(Database issue):D447-52

22. Zhou Y, Zhou B, Pache L, Chang M, Khodabakhshi AH, Tanaseichuk O, et al. Metascape provides a biologist-oriented resource for the analysis of systems-level datasets. Nature communications. 2019;10(1):1523.

23. Lai Y, Zhang F, Nayak TK, Modarres R, Lee NH, McCaffrey TA. Concordant integrative gene set enrichment analysis of multiple largescale two-sample expression data sets. BMC genomics. 2014;15 Suppl 1(Suppl 1):S6.

24. Subramanian A, Tamayo P, Mootha VK, Mukherjee S, Ebert BL, Gillette MA, et al. Gene set enrichment analysis: a knowledge-based approach for interpreting genome-wide expression profiles. Proceedings of the National Academy of Sciences of the United States of America. 2005;102(43):15545-50.

25. Zhao C, Quan X, He J, Zhao R, Zhang Y, Li X, et al. Identification of significant gene biomarkers of low back pain caused by changes in the osmotic pressure of nucleus pulposus cells. Scientific reports. 2020;10(1):3708.

26. Emmert-Streib F, Zhang SD, Hamilton P. Computational cancer biology: education is a natural key to many locks. BMC cancer. 2015;15:7.

27. Ferté C, Trister AD, Huang E, Bot BM, Guinney J, Commo F, et al. Impact of bioinformatic procedures in the development and translation of high-throughput molecular classifiers in oncology. Clinical cancer research : an official journal of the American Association for Cancer Research. 2013;19(16):4315-25.

28. Phillips KL, Cullen K, Chiverton N, Michael AL, Cole AA, Breakwell LM, et al. Potential roles of cytokines and chemokines in human intervertebral disc degeneration: interleukin-1 is a master regulator of catabolic processes. Osteoarthritis Cartilage. 2015;23(7):116577.

29. Sadowska A, Touli E, Hitzl W, Greutert H, Ferguson SJ, Wuertz-Kozak K, et al. Inflammaging in cervical and lumbar degenerated intervertebral discs: analysis of proinflammatory cytokine and TRP channel expression. European spine journal : official publication of the European Spine Society, the European Spinal Deformity Society, and the European Section of the Cervical Spine Research Society. 2018;27(3):564-77.

30. Navone SE, Marfia G, Giannoni A, Beretta M, Guarnaccia L, Gualtierotti R, et al. Inflammatory mediators and signalling pathways controlling intervertebral disc degeneration. Histology and histopathology. 2017;32(6):523-42.

31. Molinos M, Almeida CR, Caldeira J, Cunha C, Gonçalves RM, Barbosa MA. Inflammation in intervertebral disc degeneration and regeneration. Journal of the Royal Society, Interface. 2015;12(104):20141191.

32. Gabr MA, Jing L, Helbling AR, Sinclair SM, Allen KD, Shamji MF, et al. Interleukin-17 synergizes with IFNy or TNFa to promote inflammatory mediator release and intercellular adhesion molecule-1 (ICAM-1) expression in human intervertebral disc cells. Journal 
of orthopaedic research : official publication of the Orthopaedic Research Society. 2011;29(1):1-7.

33. Huang KY, Lin RM, Chen WY, Lee CL, Yan JJ, Chang MS. IL-20 may contribute to the pathogenesis of human intervertebral disc herniation. Spine (Phila Pa 1976). 2008;33(19):2034-40.

34. Smith LJ, Chiaro JA, Nerurkar NL, Cortes DH, Horava SD, Hebela NM, et al. Nucleus pulposus cells synthesize a functional extracellular matrix and respond to inflammatory cytokine challenge following long-term agarose culture. European cells \& materials. 2011;22:291-301.

35. Hoyland JA, Le Maitre C, Freemont AJ. Investigation of the role of IL-1 and TNF in matrix degradation in the intervertebral disc. Rheumatology (Oxford, England). 2008;47(6):809-14.

36. Séguin CA, Pilliar RM, Roughley PJ, Kandel RA. Tumor necrosis factor-alpha modulates matrix production and catabolism in nucleus pulposus tissue. Spine (Phila Pa 1976). 2005;30(17):1940-8.

37. Séguin CA, Pilliar RM, Madri JA, Kandel RA. TNF-alpha induces MMP2 gelatinase activity and MT1-MMP expression in an in vitro model of nucleus pulposus tissue degeneration. Spine (Phila Pa 1976). 2008;33(4):356-65.

38. Altun I. Cytokine profile in degenerated painful intervertebral disc: variability with respect to duration of symptoms and type of disease. The spine journal : official journal of the North American Spine Society. 2016;16(7):857-61.

39. Schroeder M, Viezens L, Schaefer C, Friedrichs B, Algenstaedt P, Rüther W, et al. Chemokine profile of disc degeneration with acute or chronic pain. Journal of neurosurgery Spine. 2013;18(5):496-503.

40. Wang F, Cai F, Shi R, Wang XH, Wu XT. Aging and age related stresses: a senescence mechanism of intervertebral disc degeneration. Osteoarthritis Cartilage. 2016;24(3):398-408.

41. Kepler CK, Ponnappan RK, Tannoury CA, Risbud MV, Anderson DG. The molecular basis of intervertebral disc degeneration. The spine journal : official journal of the North American Spine Society. 2013;13(3):318-30.

42. Vo NV, Hartman RA, Yurube T, Jacobs LJ, Sowa GA, Kang JD. Expression and regulation of metalloproteinases and their inhibitors in intervertebral disc aging and degeneration. The spine journal : official journal of the North American Spine Society. 2013;13(3):33141.

43. White GE, Iqbal AJ, Greaves DR. CC chemokine receptors and chronic inflammation-therapeutic opportunities and pharmacological challenges. Pharmacological reviews. 2013;65(1):47-89.

44. Beider K, Abraham M, Peled A. Chemokines and chemokine receptors in stem cell circulation. Frontiers in bioscience : a journal and virtual library. 2008;13:6820-33.

45. Ahn SH, Cho YW, Ahn MW, Jang SH, Sohn YK, Kim HS. mRNA expression of cytokines and chemokines in herniated lumbar intervertebral discs. Spine (Phila Pa 1976). 2002;27(9):911-7.

46. Pedersen LM, Schistad E, Jacobsen LM, Røe C, Gjerstad J. Serum levels of the pro-inflammatory interleukins 6 (IL-6) and -8 (IL-8) in patients with lumbar radicular pain due to disc herniation: A 12-month prospective study. Brain, behavior, and immunity. 2015;46:132-6.

47. Wang K, Bao JP, Yang S, Hong X, Liu L, Xie XH, et al. A cohort study comparing the serum levels of pro- or anti-inflammatory cytokines in patients with lumbar radicular pain and healthy subjects. European spine journal : official publication of the European Spine Society, the European Spinal Deformity Society, and the European Section of the Cervical Spine Research Society. 2016;25(5):1428-34.

48. Krock E, Millecamps M, Anderson KM, Srivastava A, Reihsen TE, Hari P, et al. Interleukin-8 as a therapeutic target for chronic low back pain: Upregulation in human cerebrospinal fluid and pre-clinical validation with chronic reparixin in the SPARC-null mouse model. EBioMedicine. 2019;43:487-500.

49. Palada V, Ahmed AS, Finn A, Berg S, Svensson Cl, Kosek E. Characterization of neuroinflammation and periphery-to-CNS inflammatory cross-talk in patients with disc herniation and degenerative disc disease. Brain, behavior, and immunity. 2019;75:60-71.

50. Burke JG, Watson RW, McCormack D, Dowling FE, Walsh MG, Fitzpatrick JM. Intervertebral discs which cause low back pain secrete high levels of proinflammatory mediators. The Journal of bone and joint surgery British volume. 2002;84(2):196-201.

51. Phillips KL, Chiverton N, Michael AL, Cole AA, Breakwell LM, Haddock G, et al. The cytokine and chemokine expression profile of nucleus pulposus cells: implications for degeneration and regeneration of the intervertebral disc. Arthritis research \& therapy. 2013;15(6):R213.

52. Zhang Y, Chee A, Shi P, Adams SL, Markova DZ, Anderson DG, et al. Intervertebral Disc Cells Produce Interleukins Found in Patients with Back Pain. American journal of physical medicine \& rehabilitation. 2016;95(6):407-15. 
53. Smith E, McGettrick HM, Stone MA, Shaw JS, Middleton J, Nash GB, et al. Duffy antigen receptor for chemokines and CXCL5 are essential for the recruitment of neutrophils in a multicellular model of rheumatoid arthritis synovium. Arthritis and rheumatism. 2008;58(7):1968-73.

54. Grespan R, Fukada SY, Lemos HP, Vieira SM, Napimoga MH, Teixeira MM, et al. CXCR2-specific chemokines mediate leukotriene B4dependent recruitment of neutrophils to inflamed joints in mice with antigen-induced arthritis. Arthritis and rheumatism. 2008;58(7):2030-40.

55. Liu X, Tian Y, Lu N, Gin T, Cheng CH, Chan MT. Stat3 inhibition attenuates mechanical allodynia through transcriptional regulation of chemokine expression in spinal astrocytes. PloS one. 2013;8(10):e75804.

56. Dawes JM, Calvo M, Perkins JR, Paterson KJ, Kiesewetter H, Hobbs C, et al. CXCL5 mediates UVB irradiation-induced pain. Science translational medicine. 2011;3(90):90ra60.

57. Xu W, Zhu M, Yuan S, Yu W. Spinal CXCL5 contributes to nerve injury-induced neuropathic pain via modulating GSK-3 phosphorylation and activity in rats. Neuroscience letters. 2016;634:52-9.

58. Zhang R, Liu Q, Peng J, Wang M, Li T, Liu J, et al. CXCL5 overexpression predicts a poor prognosis in pancreatic ductal adenocarcinoma and is correlated with immune cell infiltration. Journal of Cancer. 2020;11(9):2371-81.

59. Sandell LJ, Xing X, Franz C, Davies S, Chang LW, Patra D. Exuberant expression of chemokine genes by adult human articular chondrocytes in response to IL-1beta. Osteoarthritis Cartilage. 2008;16(12):1560-71.

60. Pattappa G, Peroglio M, Sakai D, Mochida J, Benneker LM, Alini M, et al. CCL5/RANTES is a key chemoattractant released by degenerative intervertebral discs in organ culture. European cells \& materials. 2014;27:124-36; discussion 36.

61. Grad S, Bow C, Karppinen J, Luk KD, Cheung KM, Alini M, et al. Systemic blood plasma CCL5 and CXCL6: Potential biomarkers for human lumbar disc degeneration. European cells \& materials. 2016;31:1-10.

62. Capossela S, Pavlicek D, Bertolo A, Landmann G, Stoyanov JV. Unexpectedly decreased plasma cytokines in patients with chronic back pain. Journal of pain research. 2018;11:1191-8.

63. Silva RL, Lopes AH, Guimarães RM, Cunha TM. CXCL1/CXCR2 signaling in pathological pain: Role in peripheral and central sensitization. Neurobiology of disease. 2017;105:109-16.

64. Zhang ZJ, Jiang BC, Gao YJ. Chemokines in neuron-glial cell interaction and pathogenesis of neuropathic pain. Cellular and molecular life sciences : CMLS. 2017;74(18):3275-91.

65. Chen G, Park CK, Xie RG, Berta T, Nedergaard M, Ji RR. Connexin-43 induces chemokine release from spinal cord astrocytes to maintain late-phase neuropathic pain in mice. Brain : a journal of neurology. 2014;137(Pt 8):2193-209.

66. Cao DL, Zhang ZJ, Xie RG, Jiang BC, Ji RR, Gao YJ. Chemokine CXCL1 enhances inflammatory pain and increases NMDA receptor activity and COX-2 expression in spinal cord neurons via activation of CXCR2. Experimental neurology. 2014;261:328-36.

67. Ni H, Wang Y, An K, Liu Q, Xu L, Zhu C, et al. Crosstalk between NFkB-dependent astrocytic CXCL1 and neuron CXCR2 plays a role in descending pain facilitation. Journal of neuroinflammation. 2019;16(1):1.

68. Zlotnik A, Yoshie O. The chemokine superfamily revisited. Immunity. 2012;36(5):705-16.

69. Griffith JW, Sokol CL, Luster AD. Chemokines and chemokine receptors: positioning cells for host defense and immunity. Annual review of immunology. 2014;32:659-702.

70. Zhang W, Nie L, Wang Y, Wang XP, Zhao H, Dongol S, et al. CCL20 Secretion from the Nucleus Pulposus Improves the Recruitment of CCR6-Expressing Th17 Cells to Degenerated IVD Tissues. PloS one. 2013;8(6):e66286.

71. Zhang Y, Liu L, Wang S, Zhao Y, Liu Y, Li J, et al. Production of CCL20 on nucleus pulposus cells recruits IL-17-producing cells to degenerated IVD tissues in rat models. Journal of molecular histology. 2016;47(1):81-9.

72. Yang S, Zhang F, Ma J, Ding W. Intervertebral disc ageing and degeneration: The antiapoptotic effect of oestrogen. Ageing research reviews. 2020;57:100978.

73. Song XX, Yu YJ, Li XF, Liu ZD, Yu BW, Guo Z. Estrogen receptor expression in lumbar intervertebral disc of the elderly: gender- and degeneration degree-related variations. Joint bone spine. 2014;81(3):250-3.

74. Wei A, Shen B, Williams LA, Bhargav D, Yan F, Chong BH, et al. Expression and functional roles of estrogen receptor GPR30 in human intervertebral disc. The Journal of steroid biochemistry and molecular biology. 2016;158:46-55.

75. Kobayashi Y. The role of chemokines in neutrophil biology. Frontiers in bioscience : a journal and virtual library. 2008;13:2400-7.

76. Haraguchi K, Kawamoto A, Isami K, Maeda S, Kusano A, Asakura K, et al. TRPM2 contributes to inflammatory and neuropathic pain through the aggravation of pronociceptive inflammatory responses in mice. The Journal of neuroscience : the official journal of the 
Society for Neuroscience. 2012;32(11):3931-41.

77. Kowalska MA, Rauova L, Poncz M. Role of the platelet chemokine platelet factor 4 (PF4) in hemostasis and thrombosis. Thrombosis research. 2010;125(4):292-6.

78. Wang Q, Rozelle AL, Lepus CM, Scanzello CR, Song JJ, Larsen DM, et al. Identification of a central role for complement in osteoarthritis. Nature medicine. 2011;17(12):1674-9.

79. Gharbi M, Sanchez C, Mazzucchelli G, De Pauw E, Henrotin Y. Identification of differential pattern of protein expression in canine osteoarthritis serum after anterior cruciate ligament transection: a proteomic analysis. Veterinary journal (London, England : 1997). 2013;197(3):848-53.

\section{Figures}

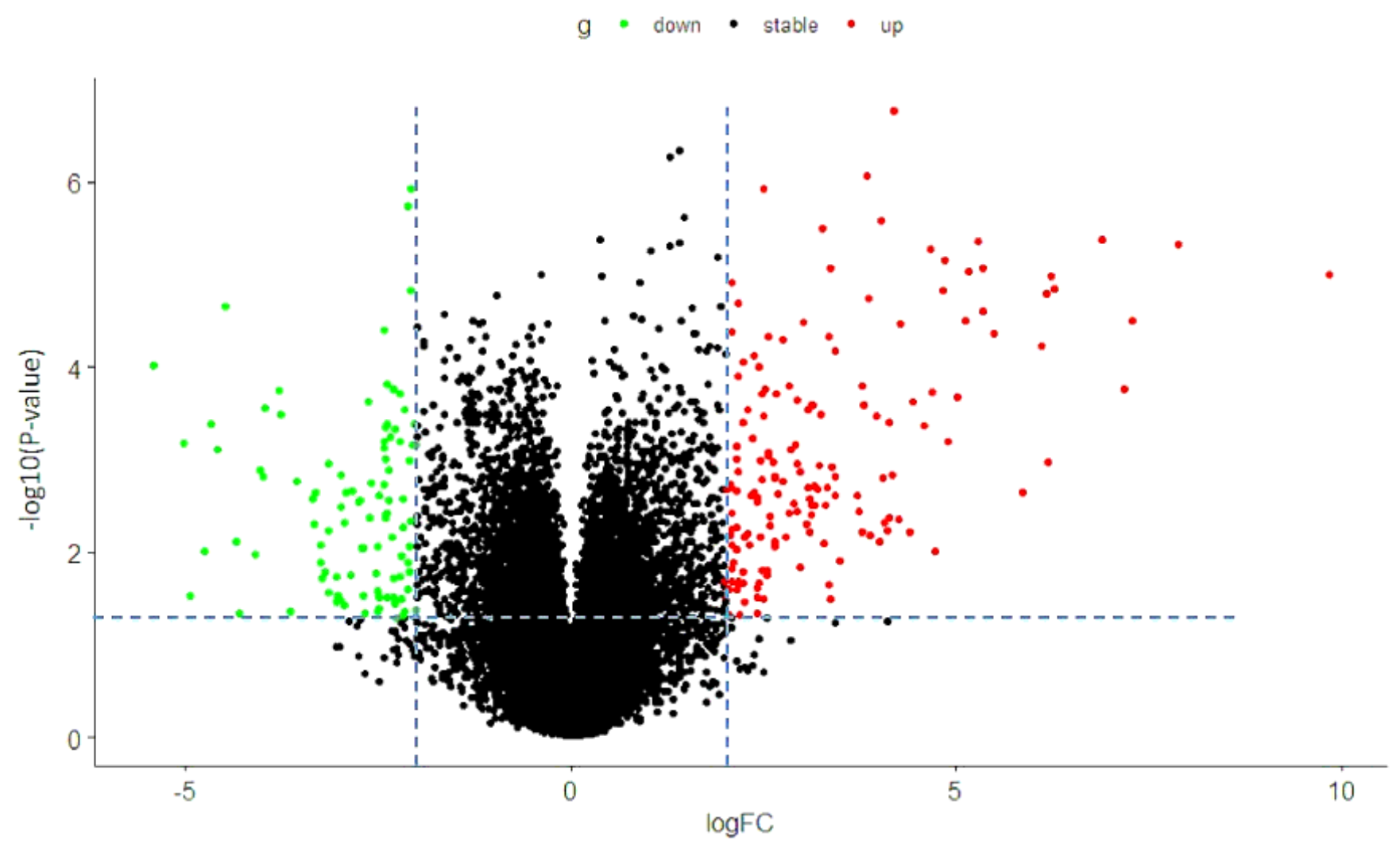

Figure 1

Volcano plot presenting the DEGs between IL-1 $\beta$-exposed and control disc cells. The down-regulated DEGs are marked in green, and the up-regulated DEGs are marked in red. 


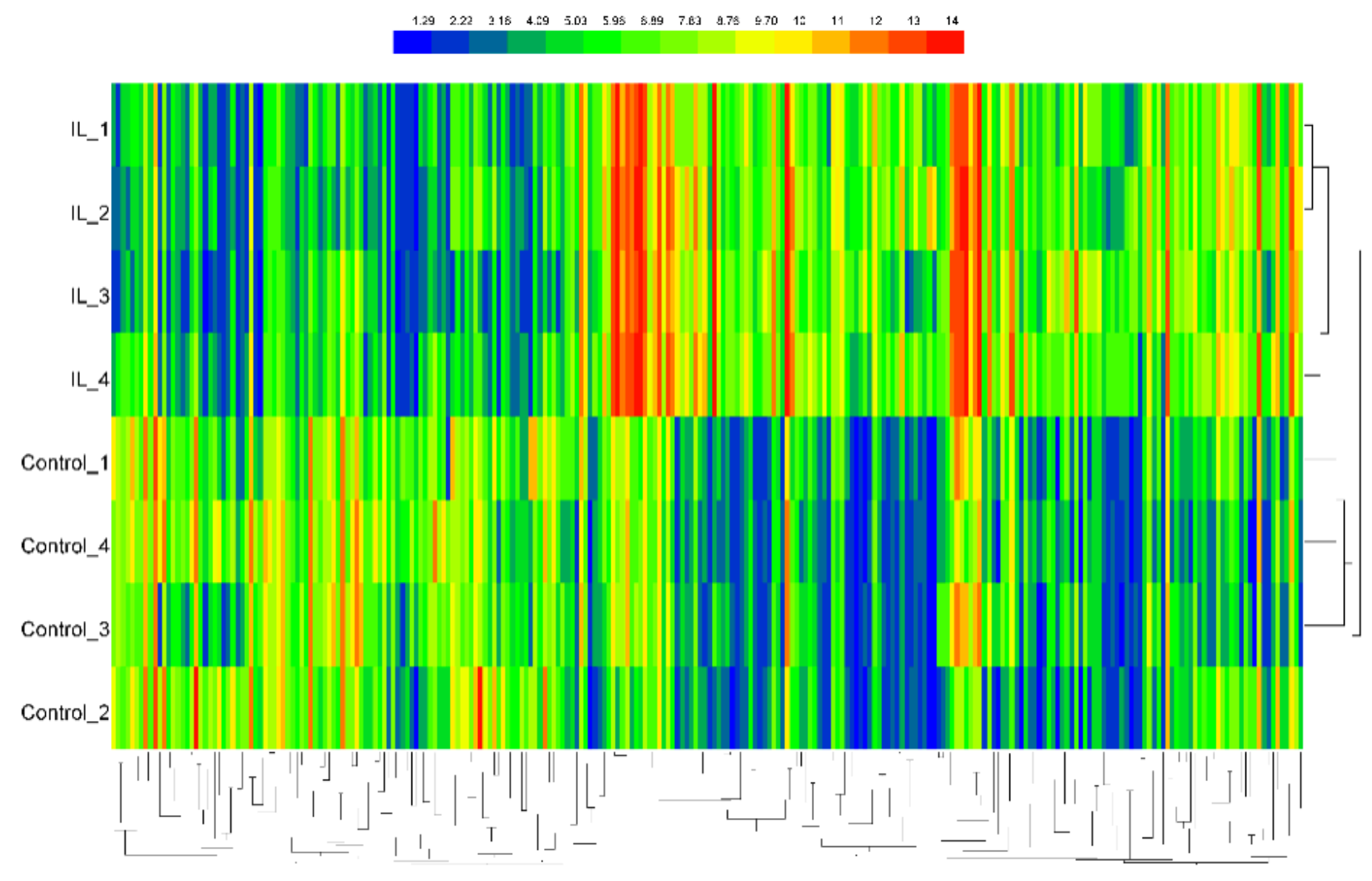

Figure 2

Heatmap of DEGs between IL-1 $\beta$-exposed and control disc cells. Each column represents a DEG and each row represents a single sample. Red color indicates an up-regulation and the green color indicates a down-regulation. 

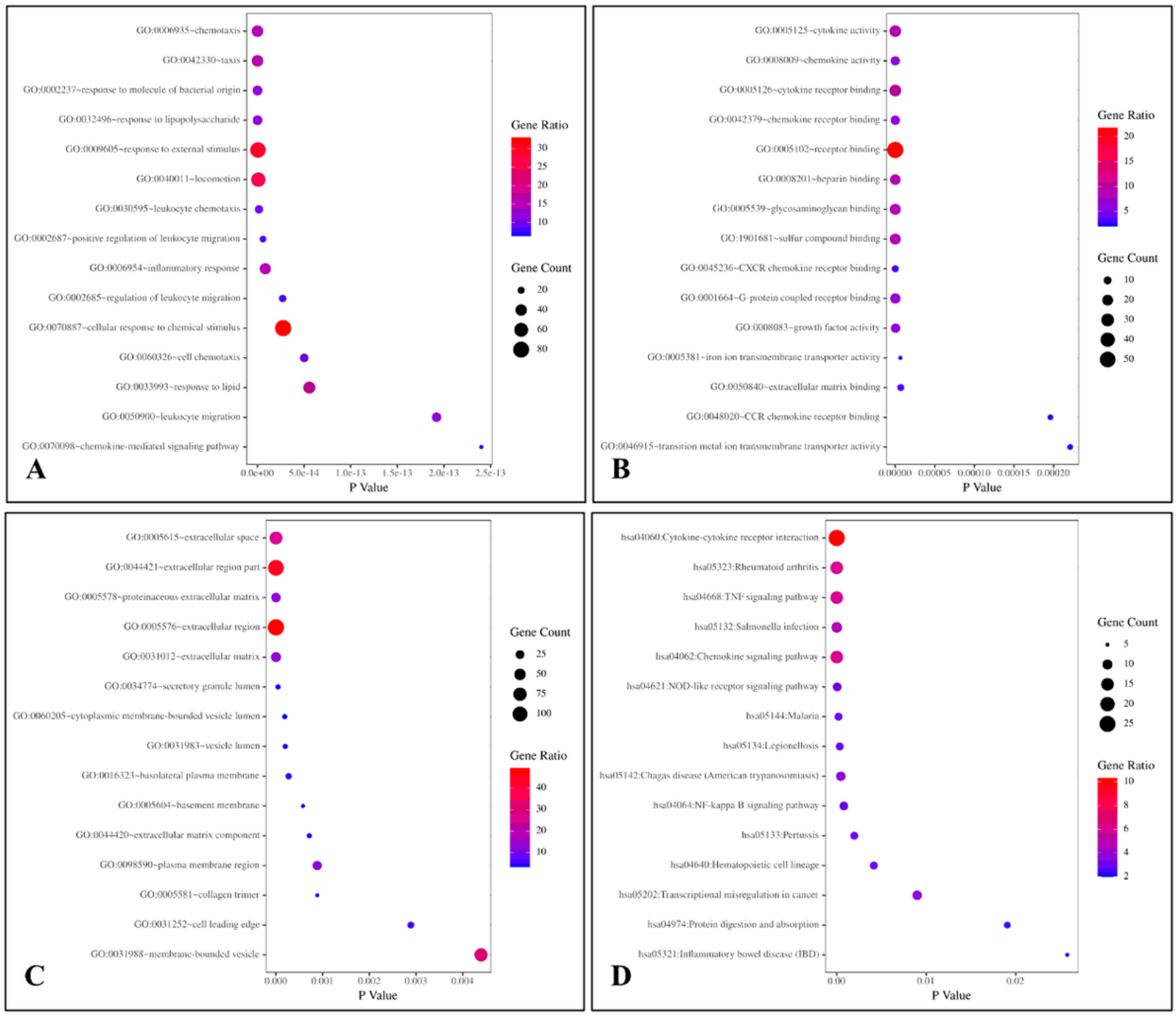

\section{Figure 3}

Bubble diagram of GO analysis and KEGG pathway analysis using DAVID. Detailed information relating to changes in the (A) biological process, (B) molecular function, (C) cellular components, and (D) KEGG analysis for hub genes. 

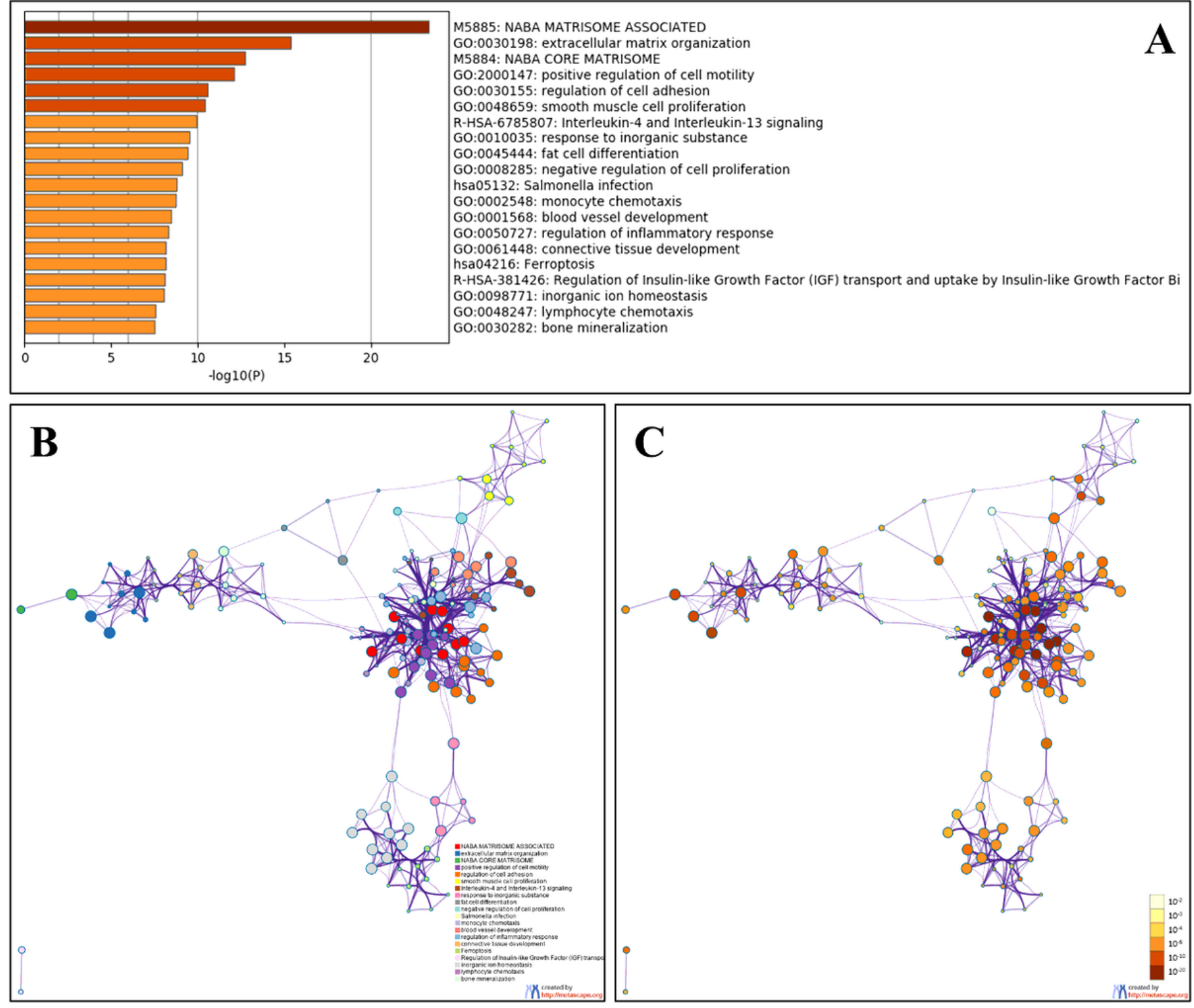

\section{Figure 4}

Enrichment analysis of DEGs using Metascape. (A) Heatmap of enriched terms across input differentially expressed gene lists, colored by p-values, obtained using Metascape. (B) Network of enriched terms colored by cluster identity, in which the nodes that share the same cluster identity are typically close to each other. (C) Network of enriched terms colored by p-value, in which the terms containing more genes tend to have a more significant $\mathrm{p}$-value. 
Enrichment plot:

GO_REGULATION_OF_INOSITOL_PHOSPHATE_BIOSYN THETIC_PROCESS

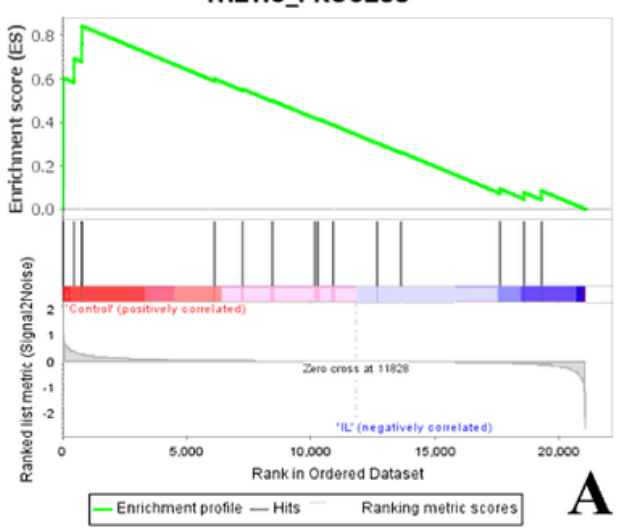

Enrichment plot: GO_NEUTROPHIL_MIGRATION

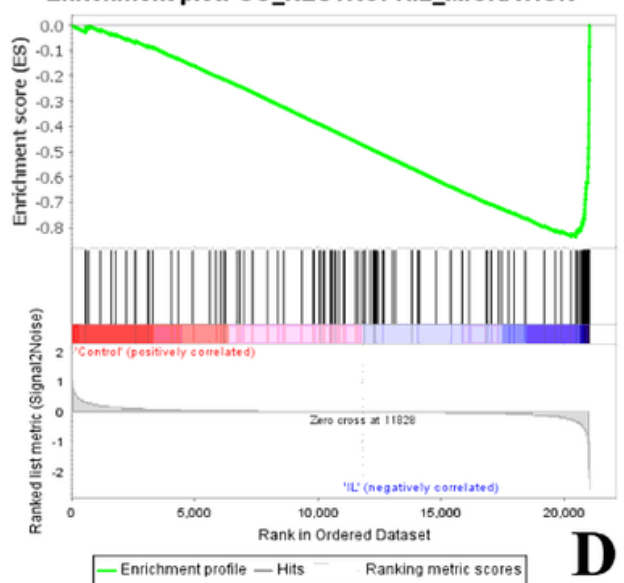

Enrichment plot:

GO_IMMUNOGLOBULIN_RECEPTOR_BINDING

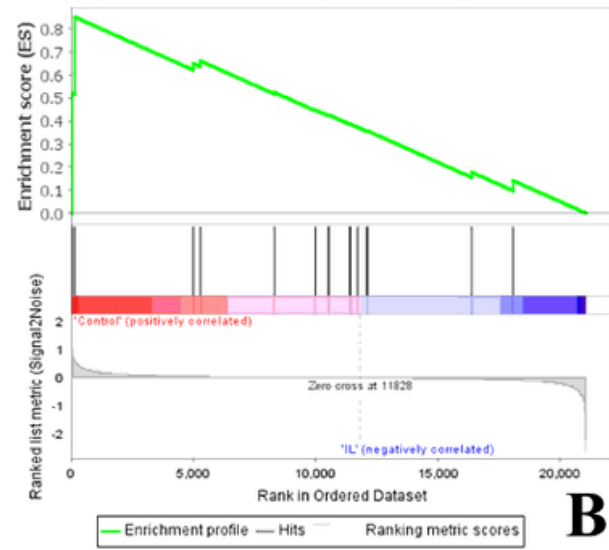

Enrichment plot:

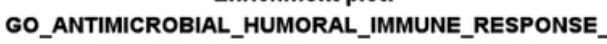
MEDIATED_BY_ANTIMICROBIAL_PEPTIDE

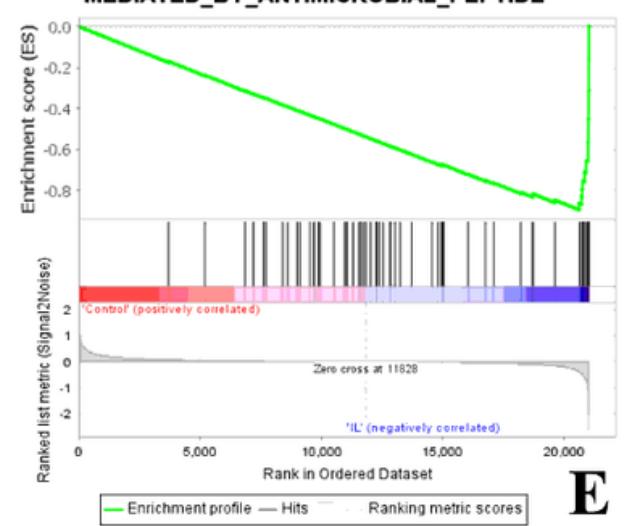

Enrichment plot: GO_WATER_TRANSPORT

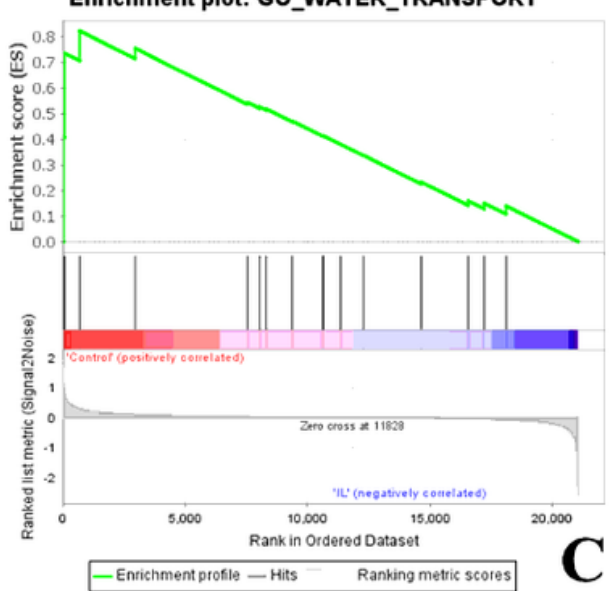

Enrichment plot: GO_GRANULOCYTE_MIGRATION

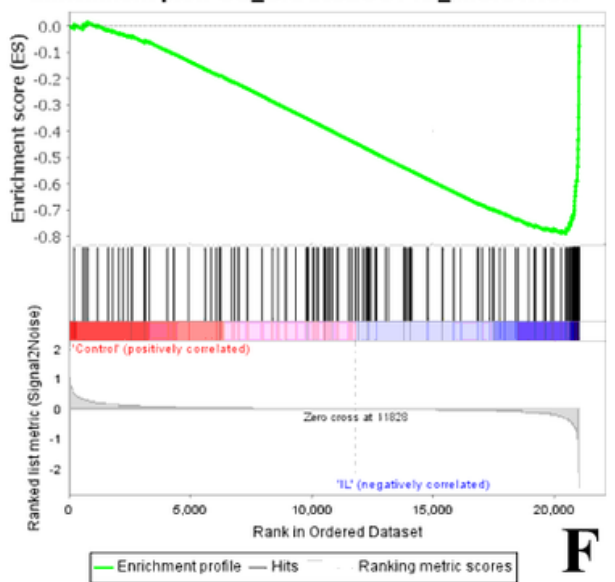

Figure 5

Six significant enrichment plots of functional enrichment analysis of DEGs between IL-1 $\beta$-exposed and control samples in GSE27494 using GSEA. (A) Enrichment plot: GO_REGULATION_OF_INOSITOL_PHOSPHATE_BIOSYNTHETIC_PROCESS. Profile of the Running ES Score \& Positions of Gene Set Members on the Rank Ordered List. (B) Enrichment plot: GO_IMMUNOGLOBULIN_RECEPTOR_BINDING. Profile of the Running ES Score \& Positions of Gene Set Members on the Rank Ordered List. (C) Enrichment plot: GO_WATER_TRANSPORT. Profile of the Running ES Score \& Positions of Gene Set Members on the Rank Ordered List. (D) Enrichment plot: GO_NEUTROPHIL_MIGRATION. Profile of the Running ES Score \& Positions of Gene Set Members on the Rank Ordered List. (E) Enrichment plot: GO_ANTIMICROBIAL_HUMORAL_IMMUNE_RESPONSE_MEDIATED_BY_ANTIMICROBIAL_PEPTIDE. Profile of the Running ES Score \& Positions of Gene Set Members on the Rank Ordered List. (F) Enrichment plot: GO_GRANULOCYTE_MIGRATION. Profile of the Running ES Score \& Positions of Gene Set Members on the Rank Ordered List. 


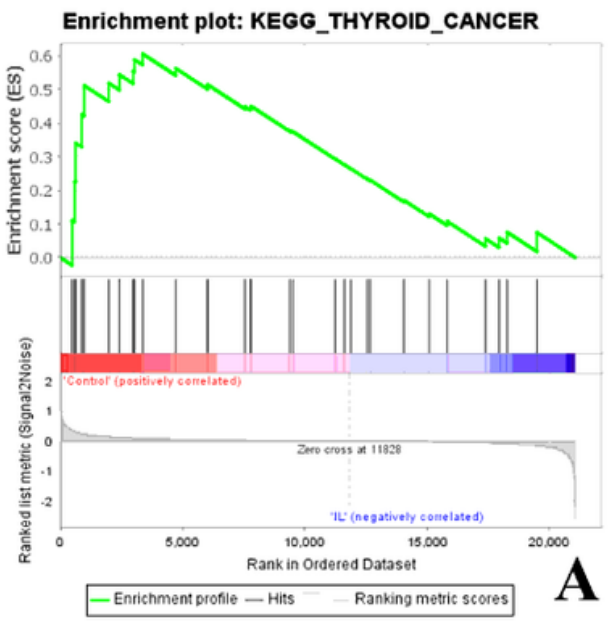

Enrichment plot: KEGG_BASE_EXCISION_REPAIR

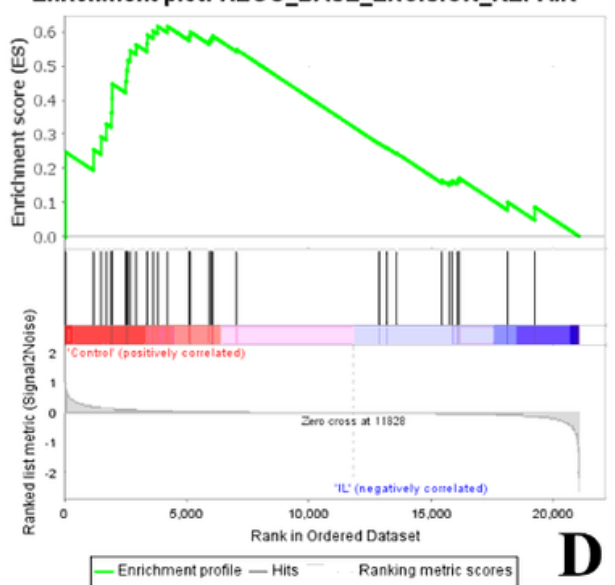

Enrichment plot: KEGG_DILATED_CARDIOMYOPATHY

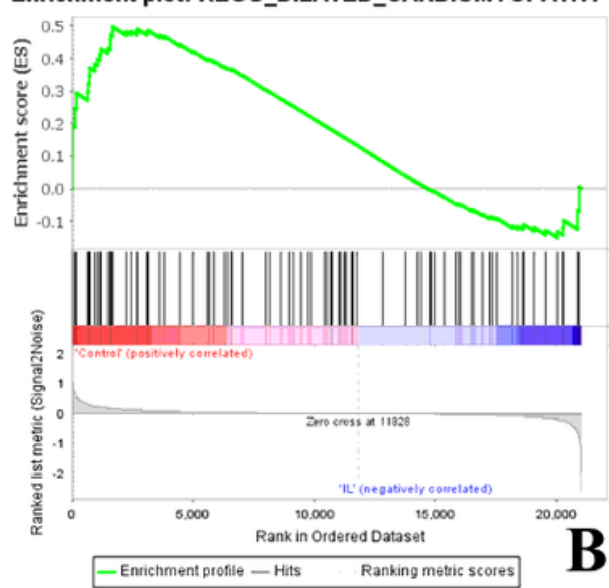

Enrichment plot: KEGG_CHEMOKINE_SIGNALING_PATHWAY

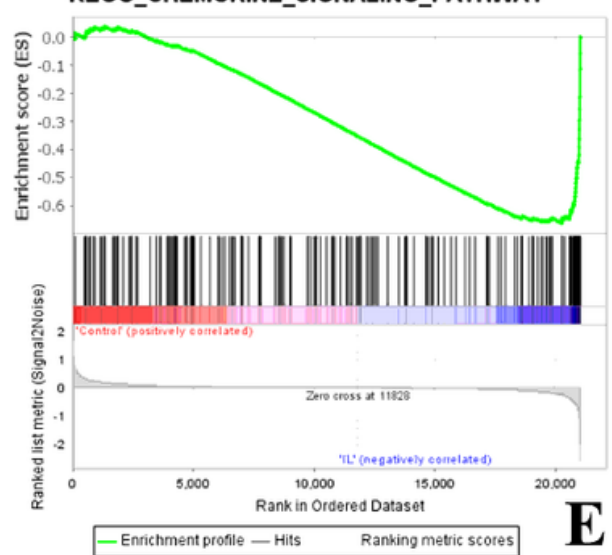

Enrichment plot: KEGG_CYTOKINE_CYTOKINE_RECEPTOR_INTERACTIO $\mathbf{N}$

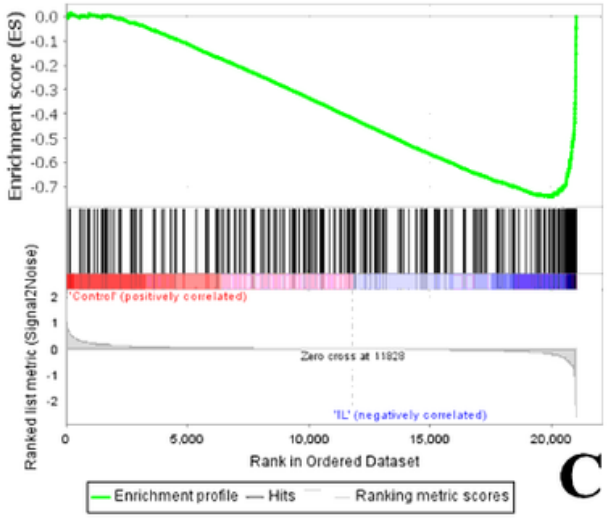

Enrichment plot: KEGG_GRAFT_VERSUS_HOST_DISEASE

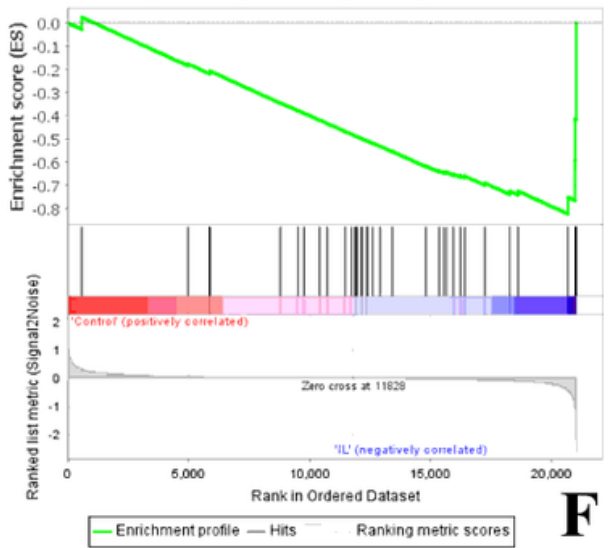

Figure 6

Six significant enrichment plots of pathway enrichment analysis of DEGs between IL-1ß-exposed and control samples in GSE27494 using GSEA. (A) Enrichment plot: KEGG_BASE_EXCISION_REPAIR. Profile of the Running ES Score \& Positions of Gene Set Members on the Rank Ordered List. (B) Enrichment plot: KEGG_THYROID_CANCER. Profile of the Running ES Score \& Positions of Gene Set Members on the Rank Ordered List. (C) Enrichment plot: KEGG_DILATED_CARDIOMYOPATHY. Profile of the Running ES Score \& Positions of Gene Set Members on the Rank Ordered List. (D) Enrichment plot: KEGG_CYTOKINE_CYTOKINE_RECEPTOR_INTERACTION. Profile of the Running ES Score \& Positions of Gene Set Members on the Rank Ordered List. (E) Enrichment plot:

KEGG_CHEMOKINE_SIGNALING_PATHWAY. Profile of the Running ES Score \& Positions of Gene Set Members on the Rank Ordered List. (F) Enrichment plot: KEGG_GRAFT_VERSUS_HOST_DISEASE. Profile of the Running ES Score \& Positions of GeneSet Members on the Rank Ordered List. 


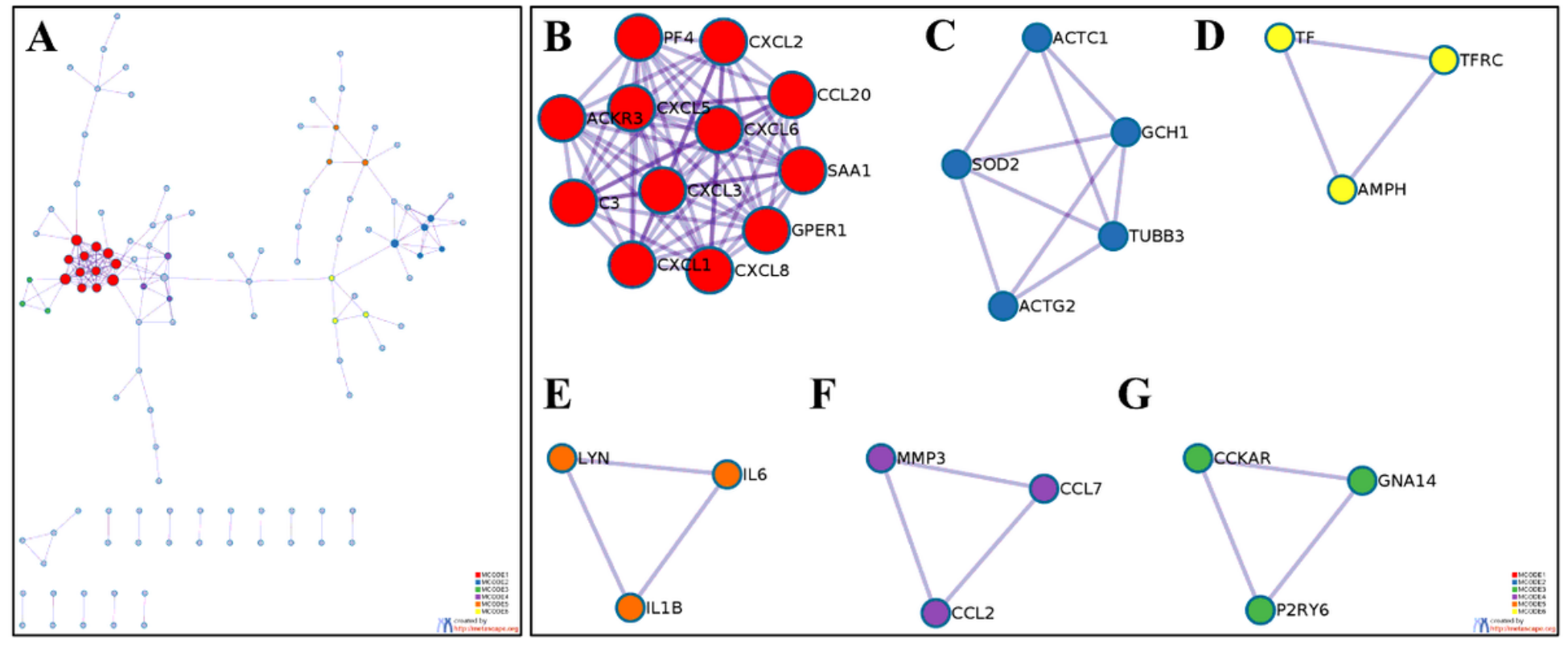

Figure 7

PPI network and significant module constructed using Metascape. (A) Through Metascape analysis, a protein-protein interaction network of DEGs was constructed. (B) MCODE 1 consists of 12 genes: CXCL2, CCL20, SAA1, GPER1, CXCL8, CXCL1, C3, ACKR3, PF4, CXCL5, CXCL3, and CXCL6. (C) MCODE 2 consists of 5 genes: ACTC1, GCH1, TUBB3, ACTG2, and SOD2. (D) MCODE 3 consists of 3 genes: TF, TFRC, and AMPH. (E) MCODE 4 consists of 3 genes: LYN, IL6, and IL1B. (F) MCODE 5 consists of 3 genes: MMP3, CCL7, and CCL2. (G) MCODE 6 consists of 3 genes: CCKAR, GNA14, and P2RY6.

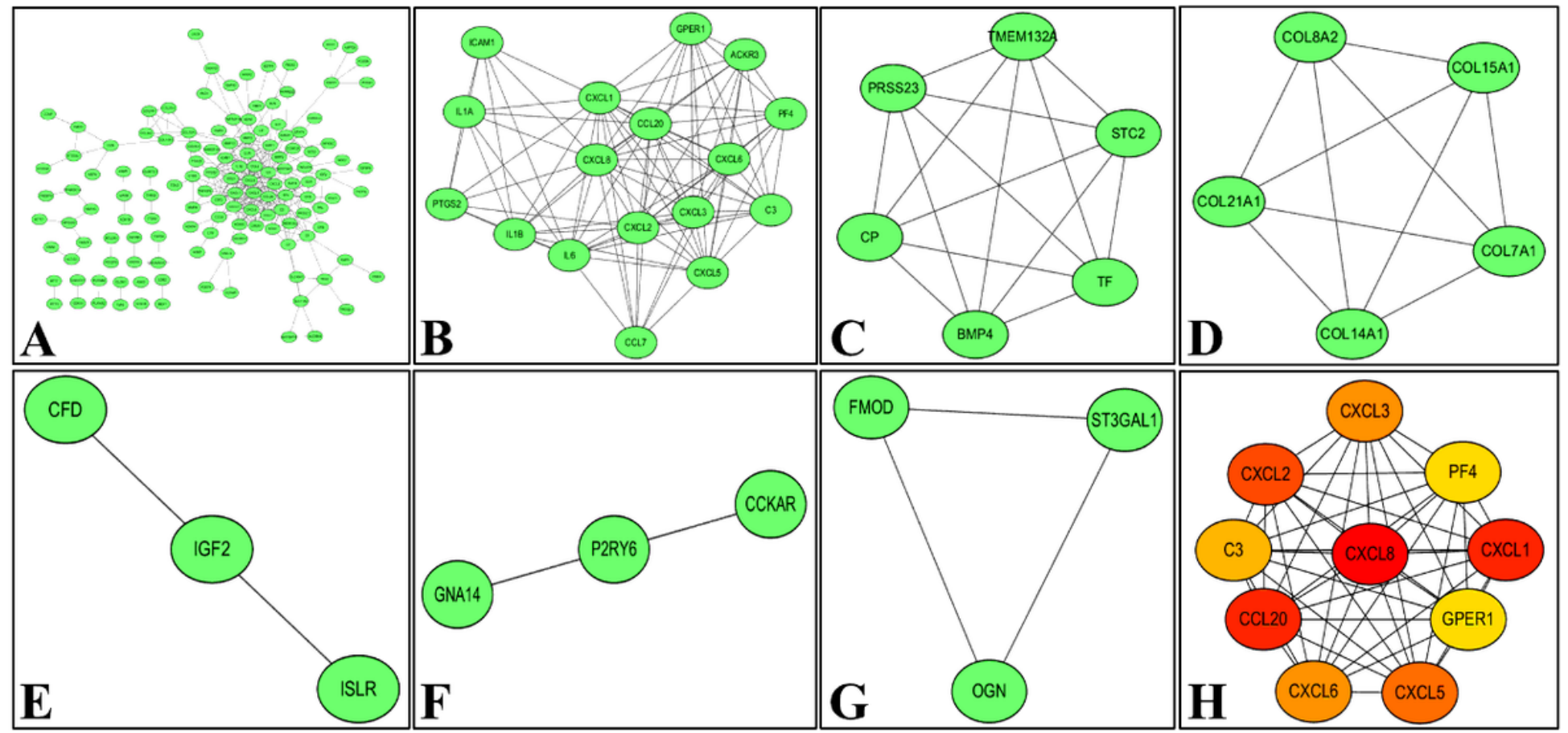

Figure 8

PPI network, significant module, and hub gene network constructed using STRING and Cytoscape. (A) There were 327 edges and 251 nodes in the PPI network. All nodes with a combined score $>0.7$ are shown. Disconnected nodes in the network were excluded. (B) The first module consists of 95 edges and 17 nodes. (C) The second module consists of 15 edges and 6 nodes. (D) The third module consists of 10 edges and 5 nodes. (E) The fourth module consists of 3 edges and 3 nodes. (F) The fifth module consists of 3 edges and 3 nodes. (G) The sixth module consists of 3 edges and 3 nodes. $(\mathrm{H})$ A total of 10 genes were identified as hub genes using Cytoscape: CXCL8, CXCL1, CCL20, CXCL2, CXCL5, CXCL3, CXCL6, C3, PF4, and GPER1. 


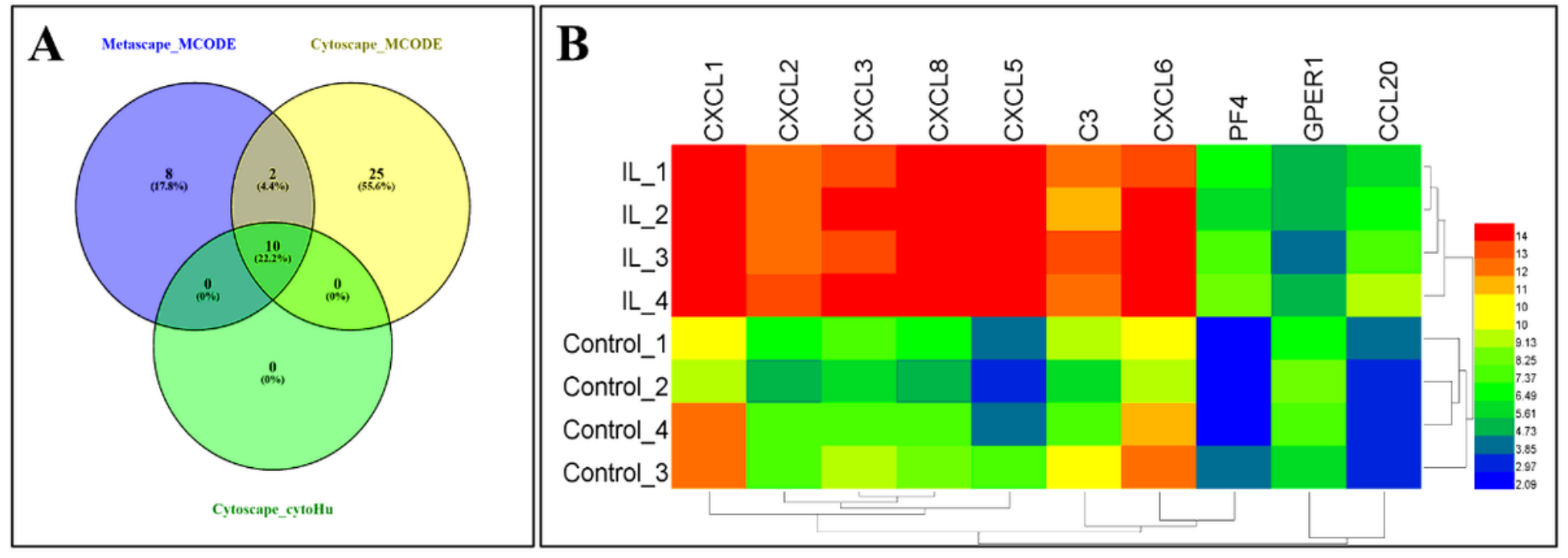

\section{Figure 9}

Identification and analysis of significant genes. (A) The VENN diagram shows that there were ten significant common genes among "Metascape_MCODE," “Cytoscape_MCODE," and "Cytoscape_cytoHubba,": CXCL8, CXCL1, CCL20, CXCL2, CXCL5, CXCL3, CXCL6, C3, PF4, and GPER1. (B) Hierarchical clustering showed that the significant genes could basically differentiate the IL-1 $\beta$-exposed disc cells from the control disc cells. 